\title{
Aspectos físico-químicos de genótipos de Passiflora alata Curtis
}

\author{
Physicochemical aspects of genotypes of Passiflora alata Curtis \\ Rafael Mesquita Borges ${ }^{1}$, Ernandes Rodrigues de Alencar ${ }^{1 *}$ (D), Ana Maria Costa ${ }^{2}$,
Nilton Tadeu Vilela Junqueira ${ }^{2}$ \\ ${ }^{1}$ Universidade de Brasília (UnB), Faculdade de Agronomia e Medicina Veterinária (FAV), Brasília/DF - Brasil
}

${ }^{2}$ Empresa Brasileira de Pesquisa Agropecuária (Embrapa), Planaltina/DF - Brasil

${ }^{*}$ Corresponding Author: Ernandes Rodrigues de Alencar, Universidade de Brasília (UnB), Faculdade de Agronomia e Medicina Veterinária (FAV), Campus Darcy Ribeiro, Asa Norte, CEP: 70910-900, Brasília/DF - Brasil, e-mail: ernandesalencar@unb.br

Cite as: Borges, R. M., Alencar, E. R., Costa, A. M., \& Junqueira, N. T. V. (2020). Physicochemical aspects of genotypes of Passiflora alata Curtis. Brazilian Journal of Food Technology, 23, e2019188.

https://doi.org/10.1590/1981-6723.18819

\begin{abstract}
Resumo
O maracujá-doce (Passiflora alata Curtis) produz frutos comercializados para consumo in natura com aroma agradável, baixa acidez e elevados teores de sólidos solúveis totais. Objetivou-se com este trabalho avaliar aspectos físicoquímicos de frutos obtidos dos diferentes genótipos de $P$. alata. Foram avaliados os frutos de 14 genótipos previamente selecionados. Avaliaram-se o rendimento de polpa com e sem sementes, a composição química, teores de potássio e de sódio, pH, acidez total titulável, teor de sólidos solúveis totais, ratio, teores de compostos fenólicos e de flavonoides e cor. $\mathrm{O}$ experimento foi delineado em blocos casualizados (DBC) com quatro repetições. Foi realizada análise de variância e posteriormente teste de Scott-Knott, a 5\% de probabilidade. Os genótipos 2, 5, 6, 7, 11, 13 e 14 se destacaram pelos maiores valores de ratio, sendo superiores a 11,0. Por outro lado, os genótipos 2, 3, 5, 7, 8, 9, 11,12 e 13 apresentaram rendimento de polpa com semente acima de $18,0 \%$. No que se refere aos compostos bioativos, destacaram-se os genótipos 1 e 7, em função dos teores de compostos fenólicos e de flavonoides, respectivamente. Os genótipos, obtidos a partir das seleções, demonstram a alta variabilidade na composição química da polpa e no conjunto de aspectos físicos e químicos do maracujá-doce.
\end{abstract}

Palavras-chave: Maracujá-doce; Qualidade; Rendimento de polpa; Composição química; Cor; Compostos bioativos.

\begin{abstract}
Sweet passion fruit (Passiflora alata Curtis) produce fruits that are marketed for in natura consumption with pleasant aroma, low acidity and high soluble solids content. The objective of this work was to evaluate the physicochemical aspects of fruits obtained from different genotypes of $P$. alata. Fruits of 14 previously selected genotypes were evaluated. The pulp yield with and without seeds, chemical composition, potassium and sodium contents, pH, total titratable acidity, total soluble solids content, ratio, total phenolic and flavonoid contents and color were evaluated. The experiment was a randomized complete block design (RCBD) with four replicates. The variance analysis and the Scott-Knott test were performed at 5\% probability. The genotypes 2, 5, 6, 7, 11, 13 and 14 were highlighted by the highest ratio values, being higher than 11.0. On the other hand, genotypes $2,3,5,7,8,9,11,12$ and 13 presented pulp
\end{abstract}


yield with seed higher than $18.0 \%$. As regards bioactive compounds, genotypes 1 and 7 were highlighted, as a function of the total phenolic and flavonoid contents, respectively. The genotypes, obtained from the selections, demonstrate the high variability in the chemical composition of the pulp and in the physical and chemical aspects of sweet passion fruit.

Keywords: Sweet passion fruit; Quality; Pulp yield; Chemical composition; Color; Bioactive compounds.

\section{Introdução}

A espécie Passiflora alata Curtis produz frutos comercializados para consumo in natura e suas folhas são utilizadas pela indústria farmacêutica e cosmética. Destaca-se que alguns autores avaliaram o potencial antioxidante de extratos de folhas e de frutos de $P$. alata, em virtude da associação da propriedade aos compostos com benefícios para a saúde (Zeraik et al., 2010; Colomeu et al., 2014; Figueiredo et al., 2016; Medeiros et al., 2018). É conhecida popularmente por maracujá-doce, maracujina, maracujá-de-colher e é uma das 150 espécies brasileiras do gênero Passiflora. Os frutos possuem sabor característico, aroma agradável, baixa acidez, elevados teores de sólidos solúveis totais (Cohen et al., 2008), sendo a polpa geralmente consumida com as sementes. O maracujá-doce não é utilizado para o preparo de sucos como ocorre para o maracujá-amarelo (Madalena et al., 2013). O fato se deve ao baixo rendimento da polpa e da baixa aceitação sensorial do suco, que apresenta sabor intenso e considerado enjoativo.

Ainda existem poucas variedades comerciais da espécie no mercado, o que dificulta o atendimento dos critérios de qualidade exigidos pela indústria e comércio de frutos. Em função da carência de material genético superior, essa espécie integra o programa de melhoramento genético da Embrapa, que lançou a "BRS Mel do Cerrado", primeira cultivar de maracujá-doce registrada (Empresa Brasileira de Pesquisa Agropecuária, 2019). O local de produção e os tratos culturais influenciam a qualidade dos frutos produzidos pelo maracujazeiro, sendo que fatores como irrigação, temperatura, adubação, cobertura e aeração são importantes para os aspectos quantitativos e qualitativos dos frutos de maracujá (Almeida et al., 2007; Carvalho et al., 2018).

Grande parte dos estudos já realizados está concentrado na espécie Passiflora edulis Sims f. flavicarpa (maracujá-azedo), devido à sua importância comercial. Em busca de diversidade, há grande interesse em pesquisas que enfoquem o desenvolvimento de outras espécies para melhor aproveitamento de suas potencialidades. Atualmente, há uma busca crescente da população mundial por alimentos mais saudáveis, inclusive objetivando a prevenção de inúmeras doenças causadas por maus hábitos alimentares. Nesse sentido, deve-se considerar as características terapêuticas presentes no gênero Passiflora advindas da sinergia de seus compostos bioativos, que têm uso farmacêutico para tratamentos de excitações nervosas, insônia, neurastenia, histerismo, menopausa, além de propriedades anti-helmínticas (vermífugas) (Cunha et al., 2002; Fumis \& Sampaio, 2007; Costa, 2017).

Apesar do melhoramento genético ter disponibilizado variedade com características mais próximas das expectativas do mercado, o maracujazeiro-doce é uma planta que apresenta ainda grandes obstáculos para a produção, tanto em termos de produtividade e resistência a pragas e doenças, como em termos de qualidade física dos frutos, tais como uniformidade e rendimento da polpa. Portanto, é fundamental continuar o trabalho de identificação de materiais genéticos superiores que apresentem características físico-químicas adequadas às exigências da produção e do consumidor que busca alimentos benéficos para a saúde. Neste cenário, o trabalho objetivou gerar informações referentes aos aspectos físico-químicos de diferentes genótipos de P. alata obtidos de três progênies do programa de melhoramento genético da Embrapa Cerrados. 


\section{Material e métodos}

O cultivo dos genótipos de $P$. alata foram conduzidos na Fazenda Experimental da Embrapa Cerrados, 15³6'13.02”S; 4743'17.34", Planaltina, DF. Os frutos foram colhidos semanalmente a partir de 30\% de amarelecimento da casca. A produção de frutos de cada planta foi individualizada. Inicialmente foram avaliadas 3 progênies conforme descrito na Tabela 1. Cada progênie compreendeu 24 genótipos de polinização aberta, totalizando 72 plantas. Em avaliação inicial, foram obtidos os dados dos 72 genótipos quanto à produtividade. De posse desses resultados, seguiu-se a pesquisa, analisando-se a qualidade físico-química dos 14 genótipos que apresentaram maior produtividade (dados não apresentados). Depois de colhidos, os frutos foram conservados a $-18^{\circ} \mathrm{C}$, até o momento das análises.

Tabela 1. Descrição das progênies de Passiflora alata.

\begin{tabular}{cl}
\hline PROGÊNIE & DESCRIÇÃO \\
\hline 1 & $\{(P$. quadrangularis $x$ P. alata $J)\}$ F1 x F1 $\{(P$. alata nativo do ES x P. alata tipo A. $)\}$ \\
\hline 2 & População segregante de $P$. alata cultivado no DF (N.R. Jardim) - Seleção 2 \\
\hline 3 & População segregante de $P$. alata cultivado no DF (N.R. Jardim) - Seleção 3 \\
\hline
\end{tabular}

Os frutos obtidos de cada planta foram pesados em balança semianalítica. Inicialmente, determinaram-se os rendimentos de polpa com e sem sementes. Os rendimentos de polpa foram obtidos pela razão entre a massa da polpa com ou sem sementes e a massa dos frutos e os resultados expressos em porcentagem. Em seguida, analisaram-se as seguintes variáveis: umidade, teores de proteínas, de lipídeos, de cinzas e de carboidratos, acidez total titulável (ATT), pH, teor de sólidos solúveis totais (SST), ratio (relação SST/ATT), coloração, teor de sódio, teor de potássio, teor de compostos fenólicos e teor de flavonoides.

A umidade foi determinada por método gravimétrico conforme Instituto Adolfo Lutz (2005). O teor de proteína foi determinado utilizando-se o método de Kjeldahl, método 991.22 (Association of Official Analytical Chemistry, 2002). O teor de lipídios foi obtido em extrator de gordura (Ankom ${ }^{\circledR}$ modelo XT 10), conforme American Oil Chemists' Society (2005). O teor de cinzas foi obtido com calcinação a $600{ }^{\circ} \mathrm{C}$, em mufla, seguindo o método 945.45 (Association of Official Analytical Chemistry, 2002). O teor de carboidratos em base seca foi obtido por diferença, subtraindo 100 dos teores de proteínas, de lipídeos e de cinzas, de acordo com o método 986.25 (Association of Official Analytical Chemistry, 2002). Os teores de proteínas, de lipídeos, de cinzas e de carboidratos foram expressos em base seca. A determinação dos teores de sódio e potássio foi realizada em fotômetro de chama, conforme método 956.01 (Association of Official Analytical Chemistry, 2002).

$\mathrm{O} \mathrm{pH}$ da polpa foi determinado em pHmetro previamente calibrado. ATT foi determinada utilizando-se solução de hidróxido de sódio ( $\mathrm{NaOH}$ ) 0,5 N e fenolftaleína como indicador (Instituto Adolfo Lutz, 2005), com os resultados expressos em porcentagem de ácido cítrico. O teor de sólidos solúveis totais (SST) foi determinado em refratômetro digital Atago (Modelo 1T), sendo os resultados expressos em ${ }^{\circ}$ Brix (Association of Official Analytical Chemistry, 2002). A partir dos valores obtidos de SST e ATT, foi possível a obtenção da relação SST/ATT (Ratio).

$\mathrm{Na}$ determinação do teor de compostos fenólicos, obteve-se inicialmente o extrato seguindo metodologia proposta por Larrauri et al. (1997). Depois de obtido o extrato, procedeu-se conforme Obanda et al. (1997). Em tubos de ensaio, foram adicionados: $1,0 \mathrm{~mL}$ do extrato obtido, 1,0 mL do Folin Ciocalteau (1:3 Folin Ciocalteau: água destilada), 2,0 $\mathrm{mL}$ do carbonato de sódio (20\%), 2,0 $\mathrm{mL}$ de água destilada e a mistura, homogeneizada. Efetuou-se leitura em espectrofotômetro a $700 \mathrm{~nm}$, sendo realizada depois de $30 \mathrm{~min}$ da adição dos reagentes. Os ensaios foram realizados em ambiente escuro. $\mathrm{O}$ branco da leitura foi $1,0 \mathrm{~mL}$ de 
água destilada acrescentado de todos os reagentes citados anteriormente. Os resultados foram expressos em miligramas de ácido gálico por $100 \mathrm{~g}$ de matéria seca (mg A.G. $100 \mathrm{~g}^{-1}$ ).

Para a determinação do teor de flavonoides, seguiu-se metodologia proposta por Lees \& Francis (1972). Pesaram-se 5,0 g de amostra fresca, com posterior adição de $30 \mathrm{~mL}$ de solução de álcool etílico e $\mathrm{HCl}$ 1,5 N $(85: 15 \mathrm{v} / \mathrm{v})$, com homogeneização por 1,0 min. Então a amostra foi transferida para balão volumétrico de $50,0 \mathrm{~mL}$, sendo completado o volume com a solução composta por álcool etílico e $\mathrm{HCl}$. A mistura foi armazenada sob abrigo da luz em ambiente refrigerado por um período de $16 \mathrm{~h}$. Decorrido esse período, a mistura foi filtrada e realizada a leitura em espectrofotômetro no comprimento de onda de $374 \mathrm{~nm}$. O teor de flavonoides totais foi expresso em $\mathrm{mg}$ por $100 \mathrm{~g}$ de matéria seca.

$\mathrm{Na}$ avaliação da coloração da polpa dos frutos, utilizou-se ColorQuest ${ }^{\mathrm{XE}}$ Spectrophotometer da Hunterlab, obtendo-se os valores das coordenadas $\mathrm{L}^{*}, \mathrm{a}^{*}$ e $\mathrm{b}^{*}$ do sistema Hunter. Utilizaram-se os valores das coordenadas a* e b* para obter a saturação da cor ou croma ( $\mathrm{C}^{*}$, Equação 1) e a tonalidade de cor (h*, Equação 2) (Little, 1975; Francis, 1975; McLellan et al., 1995).

$$
\mathrm{C}^{*}=\sqrt{\left(\mathrm{a}^{* 2}+\mathrm{b}^{* 2}\right)}
$$

$\mathrm{h}^{*}=\operatorname{arctang}\left(\mathrm{b}^{*} / \mathrm{a}^{*}\right)$

O delineamento estatístico foi em blocos casualizados (DBC), com quatro repetições, sendo cada repetição composta por quatro frutos. Inicialmente foi realizada análise de variância e posteriormente teste de Scott-Knott, a $5 \%$ de probabilidade. Utilizou-se a software SASM-Agri versão 8.2 para a análise estatística dos dados.

\section{Resultados e discussão}

Na Tabela 2, foram apresentados os dados de rendimento da polpa com semente e de polpa sem sementes, assim como da composição química da polpa de $P$. alata.

Houve diferença quando analisados o rendimento da polpa com semente e sem sementes dos diferentes genótipos de $P$. alata. $\mathrm{O}$ rendimento da polpa dos frutos com semente nos diferentes genótipos permaneceu na faixa entre $11,8 \%$ e $23,9 \%$. Quanto à variável rendimento de polpa com semente, podem ser destacados os genótipos $2,3,5,7,8,9,11,12$ e 13 , com rendimento igual ou superior a $18,0 \%$. Por outro lado, os genótipos 1 e 14 apresentaram rendimentos médios de polpa com sementes iguais a $12,1 \%$ e $11,8 \%$, respectivamente. No que se refere ao rendimento de polpa sem semente, obtiveram-se valores entre $5,6 \% \mathrm{e}$ 13,6\%. O genótipo 3 se destacou pelo maior rendimento de polpa sem semente, com valor médio de 13,6\%, diferenciando-se dos demais. Martins et al. (2003) analisaram diferentes populações de $P$. alata obtidas por polinização aberta e obtiveram rendimento médio de polpa com semente de $27,27 \%$, enquanto Freitas et al. (2006) analisaram o efeito de macronutrientes no maracujazeiro-doce e obtiveram rendimento de $22 \%$.

Tabela 2. Rendimento com e sem sementes e composição química da polpa de frutos de diferentes genótipos de Passiflora alata.

\begin{tabular}{cccccccc}
\hline Genótipos & $\begin{array}{c}\text { Polpa com } \\
\text { sementes } \\
\mathbf{( \% )}\end{array}$ & $\begin{array}{c}\text { Polpa sem } \\
\text { sementes } \\
\mathbf{( \% )}\end{array}$ & $\begin{array}{c}\text { Umidade } \\
\mathbf{( \% )}\end{array}$ & $\begin{array}{c}\text { Proteínas* } \\
\mathbf{( \% )}\end{array}$ & $\begin{array}{c}\text { Lipídeos* } \\
\mathbf{( \% )}\end{array}$ & $\begin{array}{c}\text { Cinzas* } \\
\mathbf{( \% )}\end{array}$ & $\begin{array}{c}\text { Carboidratos* } \\
\mathbf{( \% )}\end{array}$ \\
\hline 1 & $12,1 \pm 4,1 \mathrm{~b}$ & $5,6 \pm 2,9 \mathrm{c}$ & $84,7 \pm 0,7 \mathrm{a}$ & $1,5 \pm 0,2 \mathrm{a}$ & $7,1 \pm 1,9 \mathrm{c}$ & $3,4 \pm 0,5 \mathrm{c}$ & $87,9 \pm 2,1 \mathrm{a}$ \\
\hline 2 & $18,1 \pm 2,8 \mathrm{a}$ & $9,3 \pm 2,2 \mathrm{~b}$ & $81,8 \pm 1,0 \mathrm{~b}$ & $1,5 \pm 0,3 \mathrm{a}$ & $10,7 \pm 1,9 \mathrm{a}$ & $3,5 \pm 0,2 \mathrm{c}$ & $84,4 \pm 1,7 \mathrm{~b}$ \\
\hline 3 & $23,9 \pm 4,5 \mathrm{a}$ & $13,6 \pm 2,2 \mathrm{a}$ & $82,2 \pm 1,8 \mathrm{~b}$ & $1,2 \pm 0,5 \mathrm{~b}$ & $11,1 \pm 1,0 \mathrm{a}$ & $6,5 \pm 1,9 \mathrm{a}$ & $81,3 \pm 2,3 \mathrm{c}$ \\
\hline 4 & $16,1 \pm 1,8 \mathrm{a}$ & $7,9 \pm 2,9 \mathrm{~b}$ & $82,0 \pm 0,9 \mathrm{~b}$ & $1,5 \pm 0,1 \mathrm{a}$ & $11,1 \pm 2,1 \mathrm{a}$ & $4,3 \pm 1,0 \mathrm{c}$ & $81,7 \pm 1,6 \mathrm{c}$ \\
\hline 5 & $19,5 \pm 4,9 \mathrm{a}$ & $8,6 \pm 2,4 \mathrm{~b}$ & $79,8 \pm 1,4 \mathrm{c}$ & $1,0 \pm 0,3 \mathrm{~b}$ & $9,1 \pm 1,9 \mathrm{~b}$ & $2,2 \pm 0,8 \mathrm{c}$ & $87,8 \pm 2,8 \mathrm{a}$ \\
\hline 6 & $17,3 \pm 3,3 \mathrm{a}$ & $9,1 \pm 3,6 \mathrm{~b}$ & $77,2 \pm 0,6 \mathrm{~d}$ & $1,0 \pm 0,3 \mathrm{~b}$ & $8,8 \pm 3,5 \mathrm{~b}$ & $2,8 \pm 0,2 \mathrm{c}$ & $87,5 \pm 3,5 \mathrm{a}$ \\
\hline
\end{tabular}


Tabela 2. Continuação...

\begin{tabular}{cccccccc}
\hline Genótipos & $\begin{array}{c}\text { Polpa com } \\
\text { sementes } \\
\mathbf{( \% )}\end{array}$ & $\begin{array}{c}\text { Polpa sem } \\
\text { sementes } \\
\mathbf{( \% )}\end{array}$ & $\begin{array}{c}\text { Umidade } \\
\mathbf{( \% )}\end{array}$ & $\begin{array}{c}\text { Proteínas* } \\
\mathbf{( \% )}\end{array}$ & $\begin{array}{c}\text { Lipídeos* } \\
\mathbf{( \% )}\end{array}$ & $\begin{array}{c}\text { Cinzas* } \\
\mathbf{( \% )}\end{array}$ & $\begin{array}{c}\text { Carboidratos* } \\
\mathbf{( \% )}\end{array}$ \\
\hline 7 & $20,4 \pm 1,6 \mathrm{a}$ & $9,9 \pm 1,4 \mathrm{~b}$ & $83,8 \pm 0,7 \mathrm{a}$ & $1,8 \pm 0,1 \mathrm{a}$ & $6,5 \pm 0,6 \mathrm{c}$ & $3,5 \pm 0,2 \mathrm{c}$ & $88,3 \pm 0,7 \mathrm{a}$ \\
\hline 8 & $19,2 \pm 4,3 \mathrm{a}$ & $10,0 \pm 2,9 \mathrm{~b}$ & $81,9 \pm 0,7 \mathrm{~b}$ & $1,2 \pm 0,1 \mathrm{~b}$ & $8,4 \pm 0,4 \mathrm{~b}$ & $4,9 \pm 1,1 \mathrm{~b}$ & $85,6 \pm 0,7 \mathrm{~b}$ \\
\hline 9 & $18,0 \pm 3,5 \mathrm{a}$ & $8,6 \pm 2,5 \mathrm{~b}$ & $83,1 \pm 1,1 \mathrm{~b}$ & $1,4 \pm 0,1 \mathrm{a}$ & $9,2 \pm 2,4 \mathrm{~b}$ & $4,7 \pm 0,4 \mathrm{~b}$ & $84,8 \pm 2,6 \mathrm{~b}$ \\
\hline 10 & $17,7 \pm 4,7 \mathrm{a}$ & $8,0 \pm 2,4 \mathrm{~b}$ & $84,4 \pm 0,5 \mathrm{a}$ & $1,4 \pm 0,1 \mathrm{a}$ & $5,2 \pm 1,9 \mathrm{c}$ & $3,5 \pm 0,3 \mathrm{c}$ & $89,9 \pm 1,9 \mathrm{a}$ \\
\hline 11 & $19,5 \pm 4,8 \mathrm{a}$ & $8,3 \pm 2,1 \mathrm{~b}$ & $82,3 \pm 0,2 \mathrm{~b}$ & $1,4 \pm 0,1 \mathrm{a}$ & $4,4 \pm 0,1 \mathrm{c}$ & $3,9 \pm 0,2 \mathrm{c}$ & $90,4 \pm 0,2 \mathrm{a}$ \\
\hline 12 & $18,9 \pm 3,7 \mathrm{a}$ & $8,0 \pm 3,1 \mathrm{~b}$ & $85,4 \pm 2,6 \mathrm{a}$ & $1,6 \pm 0,3 \mathrm{a}$ & $5,7 \pm 0,4 \mathrm{c}$ & $4,2 \pm 1,7 \mathrm{c}$ & $88,6 \pm 1,7 \mathrm{a}$ \\
\hline 13 & $20,4 \pm 1,9 \mathrm{a}$ & $9,7 \pm 0,8 \mathrm{~b}$ & $81,5 \pm 0,4 \mathrm{~b}$ & $1,2 \pm 0,1 \mathrm{~b}$ & $7,8 \pm 2,0 \mathrm{~b}$ & $3,4 \pm 0,3 \mathrm{c}$ & $87,7 \pm 1,7 \mathrm{a}$ \\
\hline 14 & $11,8 \pm 2,2 \mathrm{~b}$ & $6,0 \pm 1,3 \mathrm{c}$ & $78,4 \pm 0,8 \mathrm{~d}$ & $1,1 \pm 0,1 \mathrm{~b}$ & $4,6 \pm 0,4 \mathrm{c}$ & $3,9 \pm 0,1 \mathrm{c}$ & $87,0 \pm 6,8 \mathrm{a}$ \\
\hline
\end{tabular}

*Expresso em base seca. Valores médios seguidos de mesma letra na mesma coluna não diferem estatisticamente pelo Teste de Scott-Knott a $5 \%$ de probabilidade.

Com relação à composição química dos frutos, verificou-se diferença para todas as variáveis analisadas. A umidade da polpa dos frutos dos genótipos variou entre 77,2\% e 85,4\%. Destaca-se que os genótipos 1, 7, 10 e 12 apresentaram valores médios de umidade iguais ou superiores a $83,8 \%$. Os menores valores médios de umidade foram obtidos quando se analisaram as polpas dos frutos dos genótipos 6 e 14, sendo iguais a 77,2\% e 78,4\%, respectivamente. $\mathrm{O}$ teor de proteínas permaneceu na faixa entre 1,0\% e 1,8\% em base seca. $\mathrm{O}$ genótipo 7 apresentou teor de proteínas equivalente a 1,8\%, sendo esse valor igual aos obtidos para os genótipos 1, 2, 4, $9,10,11$ e 12. No que se refere ao teor de lipídeos, obtiveram-se valores médios entre $4,4 \%$ e $11,1 \%$ em base seca, destacando-se os genótipos 2, 3 e 4, que apresentaram teores de lipídeos superiores a 10,6\%. Com relação ao teor de cinzas em base seca, os limites inferiores e superiores da faixa de variação foram $2,2 \% \mathrm{e}$ $6,5 \%$, respectivamente. Destacou-se o genótipo 3, por apresentar teor de cinzas na polpa igual a 6,5\%. Quanto ao teor de carboidratos da polpa, obtiveram-se valores superiores a $84,00 \%$, exceto quando se analisaram as polpas dos genótipos 3 e 4 , que apresentaram valores médios de $81,3 \%$ e $81,7 \%$, respectivamente.

Quando se compararam os dados obtidos referentes à composição da polpa dos frutos dos genótipos de P. alata com aqueles contidos na Tabela Brasileira de Composição de Alimentos (Universidade Estadual de Campinas, 2011) para $P$. edulis Sims f. flavicarpa, verificou-se que os diferentes genótipos de $P$. alata apresentaram menores teores de proteínas e de lipídeos e maiores teores de carboidratos. De acordo com a Tabela Brasileira de Composição de Alimentos, a polpa de P. edulis Sims f. flavicarpa possui teores de proteínas e de lipídeos e de carboidratos em base seca iguais a $11,7 \%, 12,3 \%$ e $74,9 \%$, respectivamente. Ressalta-se que todos os genótipos de $P$. alata apresentaram teores de proteínas inferiores a 2,0\%. Quanto ao teor de lipídeos, as polpas dos genótipos 2, 3 e 4 foram as que apresentaram valores médios mais próximos ao de P. edulis Sims f. flavicarpa, de acordo com a Tabela Brasileira de Composição de Alimentos (Universidade Estadual de Campinas, 2011). No que se refere ao teor de carboidratos, todos os genótipos de P. alata apresentaram maiores valores médios em relação a $P$. edulis Sims f. flavicarpa (74,9\%). Reis et al. (2018) obtiveram teores de proteínas, de lipídeos e de carboidratos iguais a 9,9\%, 2,9\% e 79,9\% em base seca em polpa de Passiflora caerulea. Verifica-se, dessa forma, que a polpa de $P$. caerulea também apresenta teor de proteínas superior ao da polpa de $P$. alata.

$\mathrm{Na}$ Tabela 3, são apresentados os dados referentes às variáveis $\mathrm{pH}$, acidez titulável (ATT), teor de sólidos solúveis totais (SST), ratio (SST/AT), de luminosidade ( $\left.\mathrm{L}^{*}\right)$, saturação de cor $\left(\mathrm{C}^{*}\right)$ e tonalidade de cor $\left(\mathrm{h}^{*}\right)$. De acordo com a análise de variância, houve variação significativa para todas as variáveis analisadas. 
Tabela 3. Valores médios de pH, acidez total titulável (ATT), teor de sólidos solúveis totais (SST), razão SST/ATT, Luminosidade ( $\left.\mathrm{L}^{*}\right)$, saturação de cor $\left(\mathrm{C}^{*}\right)$ e tonalidade de cor $\left(\mathrm{h}^{*}\right)$ em polpa de diferentes genótipos de Passiflora alata.

\begin{tabular}{cccccccc}
\hline Genótipo & $\mathbf{p H}$ & $\begin{array}{c}\text { ATT } \\
\text { (\% ácido } \\
\text { cítrico) }\end{array}$ & SST $\left({ }^{\circ}\right.$ Brix) & SST/ATT & L* & $\mathbf{C}^{*}$ & $\mathbf{h}^{*}$ \\
\hline 1 & $3,1 \pm 0,1 \mathrm{~d}$ & $1,9 \pm 0,2 \mathrm{a}$ & $16,0 \pm 0,6 \mathrm{~d}$ & $8,5 \pm 0,9 \mathrm{c}$ & $36,7 \pm 1,3 \mathrm{a}$ & $23,2 \pm 3,6 \mathrm{a}$ & $74,3 \pm 3,8 \mathrm{~d}$ \\
\hline 2 & $3,3 \pm 0,1 \mathrm{c}$ & $1,6 \pm 0,1 \mathrm{~b}$ & $18,5 \pm 0,9 \mathrm{~b}$ & $11,8 \pm 0,2 \mathrm{c}$ & $37,8 \pm 1,5 \mathrm{a}$ & $18,2 \pm 2,4 \mathrm{~b}$ & $83,4 \pm 5,2 \mathrm{~b}$ \\
\hline 3 & $3,3 \pm 0,1 \mathrm{c}$ & $1,8 \pm 0,1 \mathrm{a}$ & $18,4 \pm 0,8 \mathrm{~b}$ & $10,2 \pm 0,3 \mathrm{c}$ & $36,2 \pm 1,4 \mathrm{a}$ & $15,8 \pm 1,8 \mathrm{c}$ & $74,5 \pm 0,9 \mathrm{~d}$ \\
\hline 4 & $3,5 \pm 0,1 \mathrm{a}$ & $1,7 \pm 0,1 \mathrm{a}$ & $17,5 \pm 0,7 \mathrm{c}$ & $10,1 \pm 0,4 \mathrm{c}$ & $37,8 \pm 1,6 \mathrm{a}$ & $13,6 \pm 1,5 \mathrm{c}$ & $85,7 \pm 5,0 \mathrm{~b}$ \\
\hline 5 & $3,4 \pm 0,1 \mathrm{~b}$ & $1,3 \pm 0,2 \mathrm{c}$ & $18,8 \pm 0,8 \mathrm{~b}$ & $14,1 \pm 1,5 \mathrm{~b}$ & $34,6 \pm 1,4 \mathrm{~b}$ & $10,7 \pm 1,9 \mathrm{~d}$ & $85,0 \pm 2,8 \mathrm{~b}$ \\
\hline 6 & $3,6 \pm 0,2 \mathrm{a}$ & $1,2 \pm 0,2 \mathrm{c}$ & $21,0 \pm 0,8 \mathrm{a}$ & $17,2 \pm 1,7 \mathrm{a}$ & $33,7 \pm 1,0 \mathrm{~b}$ & $19,7 \pm 1,4 \mathrm{~b}$ & $76,2 \pm 0,5 \mathrm{~d}$ \\
\hline 7 & $3,3 \pm 0,1 \mathrm{c}$ & $1,6 \pm 0,1 \mathrm{~b}$ & $17,8 \pm 0,1 \mathrm{c}$ & $11,2 \pm 0,2 \mathrm{c}$ & $39,0 \pm 1,3 \mathrm{a}$ & $16,9 \pm 1,2 \mathrm{~b}$ & $84,1 \pm 0,7 \mathrm{~b}$ \\
\hline 8 & $3,3 \pm 0,1 \mathrm{c}$ & $1,8 \pm 0,1 \mathrm{a}$ & $18,5 \pm 0,8 \mathrm{~b}$ & $10,1 \pm 0,2 \mathrm{c}$ & $36,4 \pm 2,1 \mathrm{a}$ & $13,7 \pm 0,9 \mathrm{c}$ & $80,6 \pm 3,3 \mathrm{c}$ \\
\hline 9 & $3,3 \pm 0,1 \mathrm{c}$ & $1,8 \pm 0,2 \mathrm{a}$ & $17,1 \pm 0,9 \mathrm{c}$ & $9,9 \pm 1,1 \mathrm{c}$ & $37,7 \pm 1,7 \mathrm{a}$ & $14,9 \pm 0,7 \mathrm{c}$ & $77,2 \pm 1,9 \mathrm{~d}$ \\
\hline 10 & $3,3 \pm 0,1 \mathrm{c}$ & $1,8 \pm 0,2 \mathrm{a}$ & $17,4 \pm 2,3 \mathrm{c}$ & $9,7 \pm 0,5 \mathrm{c}$ & $32,8 \pm 1,2 \mathrm{~b}$ & $10,2 \pm 0,3 \mathrm{~d}$ & $92,8 \pm 1,9 \mathrm{a}$ \\
\hline 11 & $3,3 \pm 0,1 \mathrm{c}$ & $1,6 \pm 0,1 \mathrm{~b}$ & $17,6 \pm 0,1 \mathrm{c}$ & $11,2 \pm 0,4 \mathrm{c}$ & $38,0 \pm 0,5 \mathrm{a}$ & $18,1 \pm 0,3 \mathrm{~b}$ & $77,1 \pm 0,2 \mathrm{~d}$ \\
\hline 12 & $3,5 \pm 0,1 \mathrm{a}$ & $1,5 \pm 0,1 \mathrm{~b}$ & $15,8 \pm 0,3 \mathrm{~d}$ & $10,7 \pm 0,7 \mathrm{c}$ & $37,6 \pm 2,0 \mathrm{a}$ & $15,2 \pm 2,0 \mathrm{c}$ & $90,7 \pm 1,3 \mathrm{a}$ \\
\hline 13 & $3,4 \pm 0,1 \mathrm{~b}$ & $1,5 \pm 0,2 \mathrm{~b}$ & $18,2 \pm 0,4 \mathrm{~b}$ & $12,5 \pm 1,4 \mathrm{c}$ & $33,6 \pm 1,2 \mathrm{~b}$ & $14,8 \pm 0,4 \mathrm{c}$ & $80,8 \pm 2,8 \mathrm{c}$ \\
\hline 14 & $3,4 \pm 0,1 \mathrm{~b}$ & $1,6 \pm 0,4 \mathrm{~b}$ & $19,1 \pm 1,2 \mathrm{~b}$ & $12,3 \pm 2,3 \mathrm{c}$ & $35,7 \pm 2,0 \mathrm{a}$ & $17,6 \pm 7,5 \mathrm{~b}$ & $85,9 \pm 4,2 \mathrm{~b}$ \\
\hline
\end{tabular}

Valores médios seguidos de mesma letra na mesma coluna não diferem estatisticamente pelo Teste de Scott-Knott a 5\% de probabilidade.

$\mathrm{O}$ pH da polpa dos frutos de P. alata variou entre 3,1 e 3,6. Os genótipos 4, 6 e 12 se destacaram por apresentar os maiores valores médios de $\mathrm{pH}$, sendo iguais a 3,5, 3,6 e 3,5, respectivamente, semelhantes ao resultado obtido por Dutra et al. (2016), que obtiveram valor médio igual a 3,5. Destaca-se, entretanto, que a polpa dos frutos do genótipo 1 apresentou valor médio de pH equivalente a 3,1. Mamede et al. (2017), por outro lado, obtiveram valores de $\mathrm{pH}$ superiores aos obtidos no presente estudo em genótipos de $P$. alata, permanecendo entre 3,69 e 3,72. A variável ATT permaneceu na faixa entre 1,2\% e 1,9\%, inferior ao valor médio obtido por Damatto Junior et al. (2005), que foi de 2,21\%. Ressalta-se que a diferença mais expressiva foi observada quando se comparou o valor médio de ATT da polpa dos frutos do genótipo $1 \mathrm{com}$ aquele obtido para a polpa dos frutos do genótipo 6 .

No que se refere à variável SST, obtiveram-se valores médios entre 15,8 e $21,0^{\circ} \mathrm{Brix}$. Salienta-se o valor médio de SST obtido para a polpa do genótipo 6, equivalente a $21,0^{\circ}$ Brix. Além do genótipo 6 , também se destacaram os genótipos 2, 3, 5, 8 e 14, com valores de SST iguais ou superiores a $18,4^{\circ}$ Brix. Salienta-se que os valores de SST obtidos no presente trabalho foram semelhantes aos apresentados por Martins et al. (2003) para polpa de $P$. alata, com variação entre 15,7 e 21,0 ${ }^{\circ}$ Brix. É importante salientar o efeito da umidade da polpa nos valores de SST. Os valores médios de umidade obtidos nas polpas dos genótipos 6 e 14 foram inferiores a 79,0\% (Tabela 2), sendo que esses genótipos se destacaram pelos elevados valores de SST. Menores valores de umidade implicam maior concentração dos constituintes não aquosos.

Outra variável que é extremamente importante quando se analisa a polpa de frutos é a relação SST/ATT, que expressa a doçura relativa do produto e é utilizada para indicar a maturidade pela indústria de processamento de frutos tropicais (Askar \& Treptow, 1993). O maior valor de SST/ATT foi equivalente a 17,2, obtido na polpa dos frutos do genótipo 6. Por outro lado, a polpa dos frutos do genótipo 1 apresentou baixo valor médio de SST/ATT, sendo igual a 8,5. Ainda referente a SST/ATT, foi possível obter valores 
superiores aos apresentados por Damatto Junior et al. (2005) para polpa de frutos de $P$. alata cultivados com adubação orgânica. Esses autores obtiveram valores médios de SST/ATT entre 8,6 e 10,9. Jesus et al. (2018) avaliaram duas seleções de maracujazeiro-doce e obtiveram valores médios de SST/ATT equivalentes a 13,95 e 11,90. Por outro lado, Weber et al. (2017) obtiveram valores de SST/ATT inferiores aos observados no presente trabalho para polpa de $P$. alata, com médias entre 6,8 e 6,9.

Os valores médios luminosidade (L*) das polpas apresentaram variação entre 32,8 e 39,0. É importante destacar que maiores valores de luminosidade indicam que os produtos são mais claros (American Meat Science Association Meat, 2012). No que se refere à saturação de cor ( $\left.\mathrm{C}^{*}\right)$, destacou-se o genótipo 1 , que apresentou o maior valor médio $(23,2)$. Por outro lado, os genótipos 5 e 10 apresentaram polpas com os menores valores de saturação de cor, sendo equivalentes a 10,7 e 10,2, respectivamente. Jacomino et al. (2003) afirmaram que valores de saturação de cor próximos a zero indicam cores neutras (cinza), enquanto próximos de 60 implicam cores intensas ou vívidas. Dessa forma, a polpa dos frutos do genótipo 1 tem cor mais intensa ou vívida que a observada na polpa dos genótipos 5 e 10. Quanto à tonalidade de cor (h*), os valores médios permaneceram entre 74,3 (genótipo 1) e 92,8 (genótipo 10), sendo que valores próximos de zero tendem à cor vermelha, ao passo que quanto mais próximo de $90^{\circ}$, maior a predominância da cor amarela (Alves et al., 2008).

No que tange aos teores de potássio e de sódio, obteve-se variação quando se compararam os resultados dessas variáveis nas polpas dos frutos dos genótipos de $P$. alata (Tabela 4). Os valores médios de teor de potássio em base seca variaram entre 850,9 e $5.230,7 \mathrm{mg} 100 \mathrm{~g}^{-1}$. Não foi possível quantificar potássio na polpa dos genótipos 10 e 12, de acordo com a metodologia adotada no presente estudo. Dentre os genótipos em que foi possível a quantificação de potássio, o menor valor médio foi obtido na polpa do genótipo 7 . Destacaram-se pelo expressivo teor de potássio os genótipos 1, 2, 3, 4, 6, 8, 9 e 13, com valores médios entre $3.600,8$ e $5.230,7 \mathrm{mg} 100 \mathrm{~g}^{-1}$ em base seca, equivalentes a 550,9 e 835,1 mg $100 \mathrm{~g}^{-1} \mathrm{de}_{\text {massa fresca, }}$ respectivamente. Ressalta-se que a Organização Mundial de Saúde recomenda ingestão diária mínima de potássio de $3.510 \mathrm{mg} \mathrm{dia}^{-1}$ (World Health Organization, 2012). Tem-se ainda que para que um alimento seja considerado fonte de nutriente é necessário suprir pelo menos 15,0\% da necessidade nutricional diária em cada porção de $100 \mathrm{~g}$ de massa fresca (Brasil, 1998). Nesse sentido, as polpas dos frutos dos genótipos 1, 2, 3, 4, 6, 8, 9 e 13 podem ser consideradas fontes de potássio. Em se tratando de teor de sódio, os valores médios na polpa de $P$. alata permaneceram entre 25,0 e 177,5 $\mathrm{mg} 100 \mathrm{~g}^{-1}$ em base seca. Em polpa de $P$. edulis, Romero-Rodriguez et al. (1994) obtiveram teores de potássio e de sódio em base seca iguais a 748,2 e 28,8 mg $100 \mathrm{~g}^{-1}$, respectivamente. Reis et al. (2018) avaliaram polpa de $P$. caerulea e verificaram teores médios de potássio e de sódio iguais a 2.900 e 9,9 mg $100 \mathrm{~g}^{-1}$ em base seca.

Tabela 4. Teores de sódio (Na), de potássio (K), de compostos fenólicos e de flavonoides em polpa de diferentes genótipos de Passiflora alata.

\begin{tabular}{|c|c|c|c|c|}
\hline Genótipo & $\mathrm{K}^{*}\left(\mathrm{mg} 100 \mathrm{~g}^{-1}\right)$ & $\mathrm{Na}^{*}\left(\mathrm{mg} 100 \mathrm{~g}^{-1}\right)$ & $\begin{array}{l}\text { Compostos fenólicos* } \\
\left(\mathrm{mg} \mathrm{A.G.} 100 \mathrm{~g}^{-1}\right)\end{array}$ & $\begin{array}{l}\text { Flavonoides* } \\
\left(\mathrm{mg} 100 \mathrm{~g}^{-1}\right)\end{array}$ \\
\hline 1 & $3.600,8 \pm 1.333,9 a$ & $23,1 \pm 10,4 \mathrm{e}$ & $354,8 \pm 27,5 \mathrm{a}$ & $16,2 \pm 1,9 b$ \\
\hline 2 & $3.994,2 \pm 2.293,6 \mathrm{a}$ & $75,3 \pm 25,1 b$ & $116,0 \pm 3,9 c$ & $11,0 \pm 0,69 b$ \\
\hline 3 & $5.230,7 \pm 2.499,8 \mathrm{a}$ & $67,6 \pm 27,2 d$ & $204,6 \pm 13,5 b$ & $9,8 \pm 0,4 \mathrm{c}$ \\
\hline 4 & $3.733,5 \pm 1.420,0 \mathrm{a}$ & $25,7 \pm 4,9 \mathrm{e}$ & $214,7 \pm 62,7 b$ & $13,0 \pm 2,8 b$ \\
\hline 5 & $2.546,8 \pm 1.048,3 \mathrm{a}$ & $84,3 \pm 20,7 d$ & $163,7 \pm 12,9 \mathrm{c}$ & $8,8 \pm 0,1 \mathrm{c}$ \\
\hline 6 & $3.662,5 \pm 1.623,7 \mathrm{a}$ & $81,5 \pm 24,9 d$ & $202,7 \pm 22,2 b$ & $7,7 \pm 1,3 \mathrm{c}$ \\
\hline 7 & $850,9 \pm 656,3 c$ & $109,6 \pm 34,9 \mathrm{c}$ & $197,0 \pm 20,1 b$ & $25,1 \pm 13,2 \mathrm{a}$ \\
\hline 8 & $3.722,8 \pm 562,3 \mathrm{a}$ & $54.6 \pm 24,1 \mathrm{~d}$ & $156,7 \pm 4,8 \mathrm{c}$ & $7,6 \pm 1,0 \mathrm{c}$ \\
\hline
\end{tabular}


Tabela 4. Continuação...

\begin{tabular}{|c|c|c|c|c|}
\hline Genótipo & $K^{*}\left(\operatorname{mg~} 100 g^{-1}\right)$ & $\mathrm{Na}^{*}\left(\operatorname{mg~} 100 \mathrm{~g}^{-1}\right)$ & $\begin{array}{l}\text { Compostos fenólicos* } \\
\left(m g \text { A.G. } 100 \mathrm{~g}^{-1}\right)\end{array}$ & $\begin{array}{c}\text { Flavonoides* } \\
\left(\mathrm{mg} 100 \mathrm{~g}^{-1}\right)\end{array}$ \\
\hline 9 & $3.634,1 \pm 702,6 \mathrm{a}$ & $72,5 \pm 7,6 \mathrm{~d}$ & $240,5 \pm 62,6 b$ & $13,2 \pm 0,9 b$ \\
\hline 10 & ND & $177,5 \pm 31,2 \mathrm{a}$ & $309,1 \pm 28,7 \mathrm{a}$ & $5,9 \pm 1,1 \mathrm{c}$ \\
\hline 11 & $1.876,4 \pm 377,2 b$ & $62,5 \pm 17,7 \mathrm{~d}$ & $218,1 \pm 14,6 b$ & $15,7 \pm 2,4 \mathrm{~b}$ \\
\hline 12 & ND & $137,6 \pm 11,8 b$ & $309,2 \pm 40,9 a$ & $10,8 \pm 2,3 b$ \\
\hline 13 & $3.536,4 \pm 170,5 a$ & $47,5 \pm 9,7 \mathrm{e}$ & $157,4 \pm 40,3 \mathrm{c}$ & $7,9 \pm 1,6 \mathrm{c}$ \\
\hline 14 & $1.834,4 \pm 443,5 b$ & $100,0 \pm 10,0 \mathrm{c}$ & $118,8 \pm 42,2 \mathrm{c}$ & $11,9 \pm 3,6 b$ \\
\hline
\end{tabular}

*Expresso em base seca. Valores médios seguidos de mesma letra na mesma coluna não diferem estatisticamente pelo Teste de Scott-Knott a $5 \%$ de probabilidade. ND: Não detectado pelo método utilizado.

O teor de compostos fenólicos permaneceu na faixa entre 116,0 e 354,8 mg A.G. $100 \mathrm{~g}^{-1}$ em base seca (Tabela 4). Os genótipos 1, 10 e 12 se destacaram por apresentar os maiores teores de compostos fenólicos na polpa dos frutos, com valores superiores a 300,0 mg A.G. $100 \mathrm{~g} \mathrm{~g}^{-1} \mathrm{em}$ base seca. Por outro lado, a polpa dos frutos dos genótipos 2 e 14 apresentaram teores médios de compostos fenólicos inferiores a 120,0 mg A.G. $100 \mathrm{~g}^{-1}$. Os genótipos 5, 8 e 13 não diferiram dos genótipos 2 e 14 quanto ao teor de compostos fenólicos. Vasco et al. (2008) obtiveram concentração de compostos fenólicos igual a $61,0 \mathrm{mg}$ A.G. $100 \mathrm{~g}^{-1}$ de massa fresca de polpa de $P$. edulis f. flavicarpa. Esse valor é superior aos valores observados no presente estudo para polpa de $P$. alata quando calculados em relação à massa fresca, em que o máximo valor médio obtido foi de 54,3 mg A.G. $100 \mathrm{~g} \mathrm{~g}^{-1}$, no genótipo 1. Ainda de acordo com Vasco et al. (2008), concentrações de compostos fenólicos inferiores a 100,0 mg A.G. $100 \mathrm{~g}^{-1}$ de massa fresca são considerados baixos.

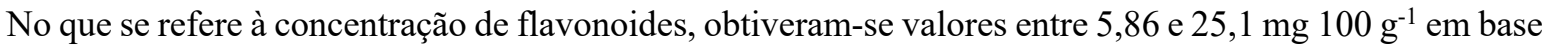
seca (Tabela 4). A polpa dos frutos do genótipo 7 se destacou por apresentar maior concentração de flavonoides $\left(25,1 \mathrm{mg} 100 \mathrm{~g}^{-1}\right)$. Por outro lado, a polpa dos frutos do genótipo 10 apresentou concentração média de flavonoides igual a $5,9 \mathrm{mg} 100 \mathrm{~g}^{-1}$.

O melhoramento de plantas faz-se a partir de seleção de características desejáveis e busca a inibição das características indesejáveis. Em trabalho de Junqueira et al. (2005), as características para o melhoramento do $P$. alata é a produção de frutos grandes com massa de 200,0 a $300,0 \mathrm{~g}$, tendo rendimento de polpa com sementes acima de $30,0 \%$ da massa fresca do fruto, alto valor nutricional e teores de sólidos solúveis totais (SST) acima de $20,0^{\circ}$.

No presente estudo, avaliaram-se diversas variáveis qualitativas de genótipos de $P$. alata pouco discutidas na literatura. Diante dos resultados obtidos, foi possível observar que o genótipo 1 apresentou maior teor de compostos fenólicos (Tabela 4), mas não se destacou quando se analisaram as variáveis rendimento de polpa e relação SST/ATT. O genótipo 6 se destacou pelo elevado valor médio de STT/ATT, equivalente a 17,2, seguido do genótipo 5, com valor médio de 14,1, mas não em termos de fenólicos e flavonoides, categoria de compostos associados a efeitos benéficos para a saúde. Mamede et al. (2017) afirmaram que os frutos de $P$. alata podem ser consumidos não apenas in natura, uma vez que os altos teores de açúcar e de sólidos solúveis associados à baixa acidez podem ser uma alternativa promissora para a produção de sucos mistos naturalmente doces.

Salienta-se que para a espécie P. alata, citada na Farmacopeia Brasileira (Brasil, 2010), a quantificação dos compostos bioativos é importante para o uso fitoterápico. Como já destacado, a polpa do genótipo 1 apresentou os maiores teores de compostos fenólicos. Em seguida, destacaram-se os genótipos 10 e 12, que não diferiram do genótipo 1 . O genótipo 7 apresentou valor médio de teor de flavonoides equivalente a $25,11 \mathrm{mg} 100 \mathrm{~g}^{-1}$, diferente dos valores obtidos para os demais genótipos. Ressalta-se que o genótipo 7 foi o que apresentou o segundo maior rendimento de polpa com semente. 


\section{Conclusão}

Os genótipos, obtidos a partir das seleções, demonstram a alta variabilidade na composição química da polpa e no conjunto de características físicas e químicas do maracujá-doce (Passiflora alata Curtis).

Os genótipos 2, 5, 6, 7, 11, 13 e 14 se destacaram por valores de ratio (SST/AT) superiores a 11,0, enquanto os genótipos 2, 3, 5, 7, 8, 9, 11, 12 e 13 possuem média de rendimento de polpa com semente igual ou superior a $18,0 \%$. Os compostos bioativos presentes na polpa dos frutos foram destaques nos genótipos 1,10 e 12 pelo teor de compostos fenólicos e, no genótipo 7, pelo teor de flavonoides.

\section{Agradecimentos}

À Coordenação de Aperfeiçoamento de Pessoal de Nível Superior (CAPES), pela bolsa de estudos, e ao CNPq, pelo custeio da pesquisa.

\section{Referências}

Almeida, E. L. P., Damatto Junior, E. R., \& Leonel, S. (2007). Nutrição mineral e adubação. In S. Leonel \& A. C. Sampaio (Eds.), Maracujádoce: Aspectos técnicos e econômicos (pp. 59-71). São Paulo: Editora UNESP.

Alves, C. C. O., Resende, J. V., Cruvinel, R. S. R., \& Prado, M. E. T. (2008). Estabilidade da microestrutura e do teor de carotenóides de pós obtidos da polpa de pequi (Caryocar brasiliense Camb.) liofilizada. Food Science and Technology, 28, 830-839. http://dx.doi.org/10.1590/S0101-20612008000400011

American Meat Science Association Meat - AMSA. (2012). Meat color measurement guidelines. Savoy: American Meat Science Association.

American Oil Chemists' Society - AOCS. (2005). Rapid determination of oil fat utilizing high temperature solvent extraction. Chicago: AOCS. Retrieved in 2019, July 5, from http://www.academia.edu/30938058

Askar, A., \& Treptow, H. (1993). Quality assurance in tropical fruit processing. Berlin: Springer Science \& Business Media. http://dx.doi.org/10.1007/978-3-642-77687-8.

Association of Official Analytical Chemistry - AOAC. (2002). Official methods of analysis of the Association of Analytical Chemistry. Arlington, Virginia: AOAC.

Brasil. Ministério da Saúde. (1998, janeiro 16). Aprova o Regulamento Técnico referente à Informação Nutricional Complementar (declarações relacionadas ao conteúdo de nutrientes) (Portaria n² 27, de 13 de janeiro de 1998). Diário Oficial [da] República Federativa do Brasil, Brasília.

Brasil. (2010). Farmacopeia brasileira (5a ed.). Brasilia: Agência Nacional de Vigilância Sanitária.

Carvalho, M. V. O., Oliveira, L. L., \& Costa, A. M. (2018). Effect of training system and climate conditions on phytochemicals of Passiflora setacea, a wild Passiflora from Brazilian savannah. Food Chemistry, 266, 350-358. PMid:30381197. http://dx.doi.org/10.1016/j.foodchem.2018.05.097

Cohen, K. O., Paes, N. S., Costa, A. M., Tupinambá, D. D., Sousa, H. N., Campos, A. V. S., Santos, A. L. B., Silva, K. N., Faleiro, F. G., \& Faria, D. A. (2008, Outubro 12-17). Características físico-químicas e compostos funcionais da polpa da Passiflora alata. In F. G. Faleiro, \& A. L. Farias Neto (Eds.), Anais do IX Simpósio Nacional sobre o Cerrado e Il Simpósio Internacional sobre Savanas Tropicais (pp. 1-6). Brasilia: Embrapa Cerrados.

Colomeu, T. C., Figueiredo, D., Cazarin, C. B. B., Schumacher, N. S. G., Maróstica Junior, M. R., Meletti, L. M. M., \& Zollner, R. L. (2014). Antioxidant and anti-diabetic potential of Passiflora alata Curtis aqueous leaves extract in type 1 diabetes mellitus (NOD-mice). International Immunopharmacology, 18(1), 106-115. PMid:24269180. http://dx.doi.org/10.1016/j.intimp.2013.11.005

Costa, A. M. (2017). Propriedade das passifloras como medicamento e alimento funcional. In T. G. Junghans, \& O. N. Jesus (Eds). Maracujá do cultivo à comercialização (pp. 299-318). Brasília: Embrapa Cerrados.

Cunha, M. A. P., Barbosa, L. C., \& Junqueira, N. T. V. (2002). Espécies de maracujazeiro. In A. A. Lima (Ed.). Maracujá produção: Aspectos técnicos (pp. 15-28). Cruz das Almas: Embrapa Mandioca e Fruticultura.

Damatto Junior, E. R., Leonel, S., \& Pedroso, C. J. (2005). Adubação orgânica na produção e qualidade de frutos de maracujá-doce. Revista Brasileira de Fruticultura, 27(1), 188-190. http://dx.doi.org/10.1590/S0100-29452005000100051

Dutra, G. A. P., Carvalho, A. J. C., Freitas, M. S. M., Santos, P. C., Freitas, J. A. A., \& Marinho, C. S. (2016). Sweet passion fruit yield and fruit quality related to fertilization with urea and cattle manure. Journal of Plant Nutrition, 39(6), 828-834. http://dx.doi.org/10.1080/01904167.2015.1109100

Empresa Brasileira de Pesquisa Agropecuária - EMBRAPA. (2019, July 3). Maracujá doce - BRS Mel do Cerrado (BRS MC). Brasília. Retrieved in 2019, July 5, from https://www.embrapa.br/busca-de-solucoes-tecnologicas/-/produto-servico/4126/maracuja-doce---brs-meldo-cerrado-brs-mc

Figueiredo, D., Colomeu, T. C., Schumacher, N. S. G., Stivanin-Silva, L. G., Cazarin, C. B. B., Meletti, L. M. M., Fernandes, L. G. R., Prado, M. A., \& Zollner, R. L. (2016). Aqueous leaf extract of Passiflora alata Curtis promotes antioxidant and anti-inflammatory effects and consequently preservation of NOD mice beta cells (non-obese diabetic). International Immunopharmacology, 35, 127-136. PMid:27039211. http://dx.doi.org/10.1016/j.intimp.2016.03.031 
Francis, F. J. (1975). The origin of tan-1 a/b. Journal of Food Science, 40(2), 412-412. http://dx.doi.org/10.1111/j.1365-2621.1975.tb02214.x

Freitas, M. S. M., Monnerat, P. H., Pinho, L. D. R., \& Carvalho, A. D. (2006). Deficiência de macronutrientes e boro em maracujazeiro doce: Qualidade dos frutos. Revista Brasileira de Fruticultura, 28(3), 492-496. http://dx.doi.org/10.1590/S0100-29452006000300033

Fumis, T. F., \& Sampaio, A. C. (2007). Aspectos botânicos do maracujá doce (Passiflora alata). In S. Leonel \& A. C. Sampaio (Eds.), Maracujá-doce: Aspectos técnicos e econômicos (pp. 25-29). São Paulo: Editora UNESP.

Instituto Adolfo Lutz - IAL. (2005). Métodos físico-químicos para análise de alimentos (4. ed.). Brasília: ANVISA, Ministério da Saúde.

Jacomino, A. P., Mendonça, K., \& Kluge, R. A. (2003). Armazenamento refrigerado de limões 'Siciliano' tratados com etileno. Revista Brasileira de Fruticultura, 25(1), 45-48. http://dx.doi.org/10.1590/S0100-29452003000100014

Jesus, C. A. S., Carvalho, E. V., Girardi, E. A., Rosa, R. C. C., \& Jesus, O. N. (2018). Fruit quality and production of yellow and sweet passion fruit in Northern state of São Paulo. Revista Brasileira de Fruticultura, 25(2), e968. http://dx.doi.org/10.1590/0100-29452018968

Junqueira, N. T. V., Peixoto, J. R., Brancher, A., Junqueira, K. P., \& Fialho, J. F. (2005). Melhoramento genético do maracujá-doce. In I. Manica, A. Brancher, C. Sanzonowicz, I. M. Icuma, J. L. P. Aguiar, J. A. Azevedo, M. A. S. Vasconcellos, \& N. T. V. Junqueira (Eds.). Maracujá- doce: Tecnologia de produção, pós-colheita e mercado (pp. 39-46). Porto Alegre: Cinco Continentes.

Larrauri, J. A., Rupérez, P., \& Saura-Calixto, F. (1997). Effect of drying temperature on the stabilitity of polyphenols and antioxidant activity of red grape pomace peels. Journal of Agricultural and Food Chemistry, 45(4), 1390-1393. http://dx.doi.org/10.1021/ff960282f

Lees, D. H., \& Francis, F. J. (1972). Standardization of pigment analyses in cranberries. HortScience, 7, 83-84.

Little, A. (1975). Off on a tangent. Journal of Food Science, 4O(2), 410-411. http://dx.doi.org/10.1111/j.1365-2621.1975.tb02213.x

Madalena, J. O., Costa, A. M., \& Lima, H. C. (2013). Avaliação de usos e conhecimentos de maracujás nativos como meio para definição de estratégias de pesquisa e transferência de tecnologia. Cadernos de Ciência \& Tecnologia, 30, 55-72.

Mamede, A. M. G. N., Soares, A. G., Oliveira, E. J., \& Farah, A. (2017). Volatile composition of sweet passion fruit (Passiflora alata Curtis). Joumal of Chemistry, 3497216, 1-9. http://dx.doi.org/10.1155/2017/3497216

Martins, M. R., Oliveira, J. C. D., Di Mauro, A. O., \& Silva, P. C. D. (2003). Avaliação de populações de maracujazeiro-doce (Passiflora alata Curtis) obtidas de polinização aberta. Revista Brasileira de Fruticultura, 25(1), 111-114. http://dx.doi.org/10.1590/S010029452003000100032

McLellan, M. R., Lind, L. R., \& Kime, R. W. (1995). Hue angle determinations and stastistical analysis for multiquadrant hunter L, a, b data. Joumal of Food Quality, 18(3), 235-240. http://dx.doi.org/10.1111/j.1745-4557.1995.tb00377.x

Medeiros, N. S., Almeida, D. C., Lima, J. D., Wohlemberg, M., Machado, F., Massolini, M., Agostini, F., Funchal, C., Bortolazzi, S., \& Dani, C. (2018). In vitro antioxidant activity of passion fruit (Passiflora alata) extract by different kinds of treatment on rat liver. Current Bioactive Compounds, 14(1), 21-25. http://dx.doi.org/10.2174/1573407213666161118120014

Obanda, M., Owuor, P. O., \& Taylor, S. J. (1997). Flavanol composition and caffeine content of green leaf as quality potential indicators of kenyan black teas. Journal of the Science of Food and Agriculture, 74(2), 209-215. http://dx.doi.org/10.1002/(SICI)1097-

0010(199706)74:2<209::AID-JSFA789>3.0.CO;2-4

Reis, L. C. R., Facco, E. M. P., Salvador, M., Flôres, S. H., \& Rios, A. O. (2018). Antioxidant potential and physicochemical characterization of yellow, purple and orange passion fruit. Journal of Food Science and Technology, 55(7), 2679-2691. PMid:30042584. http://dx.doi.org/10.1007/s13197-018-3190-2

Romero-Rodriguez, M. A., Vazquez-Oderiz, M. L., Lopez-Hernandez, J., \& Simal-Lozano, J. (1994). Composition of babaco, feijoa, passion-fruit and tamarillo produced in Galicia (NW Spain). Food Chemistry, 49(1), 23-27. http://dx.doi.org/10.1016/0308-8146(94)90227-5 Universidade Estadual de Campinas - UNICAMP. (2011). Tabela brasileira de composição de alimentos (4. ed.). Campinas: UNICAMP.

Vasco, C., Ruales, J., \& Kamal-Eldin, A. (2008). Total phenolic compounds and antioxidant capacities of major fruits from Ecuador. Food Chemistry, 111(4), 816-823. http://dx.doi.org/10.1016/j.foodchem.2008.04.054

Weber, D., Nachtigal, J., Barreto, C., \& Malgarim, M. (2017). Assessment of passion fruit trees genotypes in terms of fruit quality and yields in Rio Grande do Sul-Brazil. American Journal of Experimental Agriculture, 18(6), 1-8. http://dx.doi.org/10.9734/JEAl/2017/37819

World Health Organization - WHO. (2012). Guideline: Potassium intake for adults and children. Geneva: WHO.

Zeraik, M. L., Pereira, C. A., Zuin, V. G., \& Yariwake, J. H. (2010). Maracujá: Um alimento funcional? Revista Brasileira de Farmacognosia, 20(3), 459-471. http://dx.doi.org/10.1590/S0102-695X2010000300026

Financiamento: Coordenação de Aperfeiçoamento de Pessoal de Nível Superior - CAPES (Código de Financiamento 001); Conselho Nacional de Desenvolvimento Científico e Tecnológico - CNPq (Processo n 404847/2012-9). 